\title{
(2) OPEN ACCESS \\ Meibography guided intraductal meibomian gland probing using real-time infrared video feed
}

\author{
Steven L Maskin $\bullet$, Sreevardhan Alluri
}

Dry Eye and Cornea Treatment Center, Tampa, Florida, USA

\section{Correspondence to} Dr Steven L Maskin, Dry Eye and Cornea Treatment Center, Tampa, FL 33609, USA; drmaskin@tampabay.rr.com

Received 21 October 2019 Revised 30 December 2019 Accepted 13 February 2020 Published Online First 27 February 2020
Check for updates

(C) Author(s) (or their employer(s)) 2020. Re-use permitted under CC BY-NC. No commercial re-use. See rights and permissions. Published by BMJ.

To cite: Maskin SL, Alluri S. $\mathrm{Br} J$ Ophthalmol

2020;104:1676-1682.

\section{ABSTRACT \\ Purpose To evaluate use of infrared meibography video to visualise meibomian gland probing and correlate probe findings of intraductal space with meibography images.}

Methods Videos were reviewed and probe findings recorded of 996 probed gland orifices from 38 lower lids. Results 996/997 (99.9\%) of gland orifices were successfully probed with $91.8 \%$ revealing probe location. There were no false passages. 14\% (140/997) of all gland orifices showed whole gland atrophy (WGA) with $99.3 \%$ (139/140) probed to $1 \mathrm{~mm}$. Cumulative probe findings for all WGA (not differ significantly from non-WGA) showed 106 (76\%), 21 (15\%) and 12 (9\%) glands with fixed, non-fixed and no resistance (NR), respectively. Lids without WGA showed increased NR/ total glands probed while lids with WGAs $(\geq 5)$ showed increased NR/WGA compared with lids with only 1-4 WGAs ( $p=0.011, p=0.005$, respectively, Mann-Whitney $U$ test) suggesting bimodal NR profile. Visualisation of microtube placement was successfully obtained for therapeutic injections and retrieval of meibum specimens. Conclusion Video confirmed intraductal location and safety of devices. For $73 \%$ of non-WGA and $76 \%$ of WGAs as well as proximal ducts of glands with proximal atrophy, probing released fixed resistance restoring ductal integrity. A bimodal profile of NR suggests it is found with less diseased gland ducts as well as more advanced atrophic gland disease. Gland and ducts appeared flexible but not distendable while periglandular tissue appeared spongy. Visualisation of devices enables whole or localised gland therapy and meibum specimen retrieval, elegantly raising future research, therapeutic and regenerative opportunities.

\section{INTRODUCTION}

Obstructive meibomian gland dysfunction (MGD) is considered the most frequent cause of dry eye in the world. ${ }^{12}$ Periductal fibroses was observed at the slit lamp and written about in the late $1990 \mathrm{~s}^{3}$ and again in $2003,{ }^{4}$ followed by its description using confocal microscopy in 2008..$^{5}$ In 2010, the first author (SLM) revealed, for the first time in the literature, the unexpected finding during meibomian gland (MG) probing of intraductal strictures and obstruction presenting as fixed, firm, focal and unyielding resistance (FFFUR) in these patients. ${ }^{67}$ We recently reported $67 \%$ of all probed (expressible and nonexpressible) glands demonstrated FFFUR within $1 \mathrm{~mm}$ of the orifice and over $90 \%$ within $2 \mathrm{~mm}^{8}$ suggesting that expressible glands were just as likely to have occult obstruction as non-expressible glands to have obvious obstruction. We proposed that periductal fibroses contracting around and pinching in the external duct wall leads to a secondary loss of intraductal integrity from lumenal contraction and strictures while compromising meibum flow with increased intraductal pressure (IDP) and risk of gland atrophy. The resistance was relieved by using increased probing pressure to advance the probe and release tight, fixed periductal fibrotic bands, thereby restoring $M G$ intraductal integrity. For the past 10 years, MG probing has been performed at the slit lamp and under the operating microscope using colour visualisation to find entry angle into each gland. The clinician then advances the probe into the gland using tactile sensation. Once the fixed resistance is released, the probe device can move to and fro freely within the intraductal space and at times previously sequestered meibum may spontaneously self-express through the orifice along the wire probe as IDPs equilibrate. ${ }^{9} 10$

Despite the established safety of probing in all peer-reviewed published papers on probing ${ }^{6} 811-21$ it would be a significant technological breakthrough if the operator could utilise infrared (IR) meibography to simultaneously visualise glands and intraductal devices during the probing procedure analogous to CT directed procedures such as biopsy of vital organs. This study was performed to evaluate the use of real-time IR meibography with video feed to visualise meibomian gland probing (MGP) as well as meibography guided MGP utilising a colour slit lamp image to enter the gland through its natural orifice. We were interested if we could locate the image of probes during the procedure to evaluate safety as well as correlate probe finding and meibography appearance of the same gland. In addition, we evaluated the feasibility of imaging the location of procedures within the intraductal space such as microtube injection of therapeutics or retrieval of meibum specimen.

\section{MATERIALS AND METHODS \\ Study design and patient selection}

We conducted a retrospective review of IR video meibography of 996 probed glands from 38 lower lids of 26 consecutive patients captured during sessions of intraductal MGP for patients with obstructive meibomian gland dysfunction (table 1). Two additional patients (three lids) were not considered for this study due to excessive blinking leading to technical difficulties with the video. One gland with whole gland atrophy (WGA) could not be entered as its entry angle was not identified. Patients' average age was 65.6 years and male:female ratio was 5:21. Real-time 


\begin{tabular}{|c|c|c|c|}
\hline Total patients & 26 & TGP with visible probe & $914(91.8 \%)$ \\
\hline Male:female & $5: 21$ & False passages & $0 \%$ \\
\hline Age (mean) & 65.6 years & WGA visualised & 140 \\
\hline Total lids (lower) & 38 & WGA (probed) & 139 (14\% of TGP) \\
\hline $\begin{array}{l}\text { Lids with at least one } \\
\text { WGA }\end{array}$ & 24 & $\begin{array}{l}\text { Percentage of WGA with fixed } \\
\text { obstruction }\end{array}$ & $76 \%$ \\
\hline Glands visualised & 997 & Non-WGA (probed) & 857 ( $86 \%$ of TGP) \\
\hline TGP & 996 & $\begin{array}{l}\text { Percentage of all glands with } \\
\text { fixed obstruction }\end{array}$ & $73.5 \%$ \\
\hline
\end{tabular}

TGP, total glands probed; WGA, whole gland atrophy.

IR video feed with colour slit lamp imaging was obtained using the Mediworks S390L WDR FireFly Digital Slit Lamp from Eyefficient. This technology enabled visualisation of intraductal probing, microtube therapeutic injection and meibum specimen retrieval. Parameters evaluated were percentage of total probed glands with a visualised intraductal probe beyond the orifice, detection of any false passage and correlation of probe findings revealing specific gland intraductal space characteristics with corresponding meibography images. Probes and microtubes were obtained from Katena Products. All procedures adhered to the principles of the Declaration of Helsinki, and all patients provided informed consent. This study received an independent review board exempt review determination by an independent review board.

\section{Intraductal MGP, probe findings and other procedures under real-time IR meibography visualisation Meibomian gland probing}

MGP was performed as previously described by the first author. ${ }^{8}$ Briefly, one drop of topical 0.5\% tetracaine hydrochloride (Bausch and Lomb) was placed in the inferior fornix followed by placing a bandage contact lens over the cornea. Topical anaesthetic ointment consisting of $8 \%$ lidocaine with $25 \%$ jojoba anaesthetic ointment (JAO) in a petrolatum ointment base (O'Brien Pharmacy) was applied to the inferior lid margin. The eye was closed for $15 \mathrm{~min}$. A second application of JAO was applied when necessary. One additional drop of topical tetracaine was then placed in the eye. The patient was then positioned at the FireFly slit lamp and its IR camera was activated. The lower lid was everted and the colour image of the meibomian gland orifices and glands were focused through the slit lamp oculars while the monitor displayed the IR image of the glands for inspection. A $1 \mathrm{~mm}$ long stainless-steel sterile intraductal Meibomian gland probe was then inserted into each orifice, perpendicular to the lid margin using a dart throwing motion to find the angle of entry. The probe could be seen through the oculars' colour slit lamp image entering through the orifices only, while the monitor showed the IR image of the intraductal probe entering and moving to and fro within the gland duct. As the probe was moved, the video image followed the probe in real-time and sequentially into the distal, mid and proximal duct up to the full length of the $1 \mathrm{~mm}$ as well as 2 and $4 \mathrm{~mm}$ probes when used. The longer probes were used when clinically indicated or to evaluate patency of proximal central ducts in the setting of proximal acinar-ductule (A-D) atrophy (lack of A-D units proximal to distal or mid to distal glandular A-D units). At times the movement and advancement of the probe were completely directed by viewing the video monitor IR image and not looking through the slit lamp oculars. Using this meibography guided approach glands can be probed up to and not beyond a hairpin loop or an excessive bend in a severely tortuous gland. Also, when using meibography guided procedures, the probe and microtube devices could be directed at selective parts of the proximal, mid or distal glands to deliver localised therapeutics or retrieve specimens.

\section{Probe findings: FFFUR versus soft resistance}

In brief, the probe was passed through the orifice lumen and into the distal duct where there typically was fixed resistance to the probe. The resistance was characterised as FFFUR. It required additional probing force to relieve, analogous to relief of punctal fibrosis with a canalicular probe. Opening the obstruction created a tactile sensation of pressure release as well as audible sound of variable intensity, often heard by patient and physician as the tight band of contracting periductal fibroses was released and resistance gave way, allowing sudden advancement of the probe which was then able to freely pass to and fro within the duct. $^{8}$

Less commonly, a mild back pressure or 'soft' resistance (SFT) was noted which was not fixed, not firm and easily yielding. Soft resistance was felt, but allowed the probe to pass without significant additional mechanical pressure. Passing through SFT did not generate an audible sound. It can be thought of as providing 'drag' on the 'to and fro' movement of the wire probe. SFT was not focal in contrast to the FFFUR which characteristically was focal or multifocal. Infrequently, there was a lack of resistance designated as 'NR' where the probe entered and penetrated the orifice and duct without any resistance or drag.

\section{Intraductal microtube insertion}

In some cases, after removing the probe, a $1 \mathrm{~mm}$ long stainless steel microtube of 110 outer diameter (OD) was introduced into the glands to inject therapeutic dexamethasone. The tube was visualised within glands under IR video-meibography conditions to inject approximately $8 \mu \mathrm{l}$ of dexamethasone (EyeSupply). To retrieve a sample of meibum from within the intraductal space, an alternative $4 \mathrm{~mm}$ long microtube was placed through the already probed and dilated orifice and into the gland, visualised under IR meibography conditions. This microtube was made from polyimide with an approximate OD of 125 and inner diameter of 90 microns.

\section{IR video meibography}

Lower lid meibomian glands were visualised during intraductal insertion of probes and tubes, as well as during subsequent procedures including probing, microtube injection and meibum sample retrieval. Glands were evaluated for WGA, then correlated to probe findings of FFFUR or SFT or NR. WGA was identified where a gland showed a lack of defined acinar-ductule units for the full extent of the gland. Proximal atrophy, defined as a lack of A-D units proximal to distal or mid-distal gland A-D units, was also noted when present.

\section{Data collection and analysis}

The probe findings such as FFFUR, SFT and NR were recorded on a probing form (total glands probed (TGP)) and later correlated with the audio-video meibography recording (reviewed TGP form) in relation to the orifice-gland position along the lid margin. The numbers of WGA and non-WGA glands were counted manually from the meibography video feed performed just prior to MGP and correlated to earlier probe findings. The cumulative number of glands from all lids exhibiting FFFUR, SFT, NR, as well as WGA and non-WGA was converted into percentages by dividing these parameters by total 

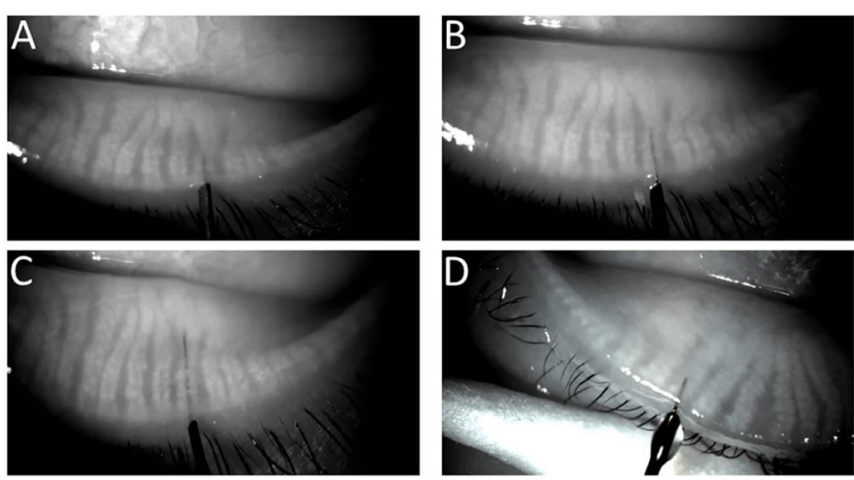

Figure 1 (A-D) Location and presence of a 1, 2 and $4 \mathrm{~mm}$ probe $(76 \mu \mathrm{m} \mathrm{OD})$ as well as a $1 \mathrm{~mm}$ tube $(110 \mu \mathrm{m}$ OD), respectively, inside the duct of a meibomian gland. Note that acinar-ductule units are visible in some areas overlying the devices.

number of probed glands and multiplying by 100 . To evaluate relationships between these probe findings and meibography appearance of WGA, the per cent FFFUR, SFT and NR within WGAs was noted for individual lids and its mean for all lids calculated. These calculations were done by taking the number of glands with the probe finding in association with WGA in a lid, divided by total WGA probed within that lid and multiplied by 100. The mean values for all lids were then calculated. FFFUR, SFT and NR were also calculated as a per cent of total glands (WGA plus non-WGA) probed for a single lid and its mean for all lids calculated. These calculations were done by taking the number of glands with the probe finding within an individual lid, divided by TGP in that lid and multiplied by 100 . All data were electronically entered for further data analysis. Patient lids were categorised into three groups based on numbers of WGA per lid. Groups 1, 2 and 3 had zero, 1-4 and $\geq 5$, respectively. IBM SPSS software V.25.0 and GraphPad Prism 7 were used for performing basic demographic statistics and non-parametric analysis to explore the statistical distribution of probe findings
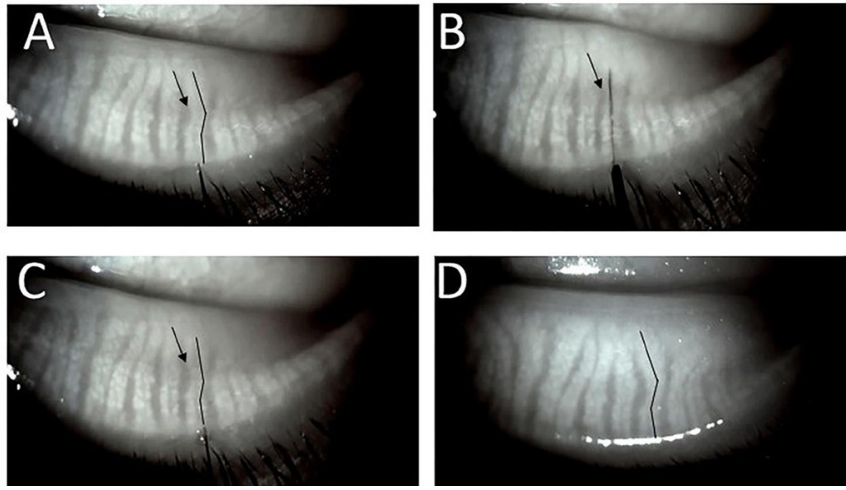

Figure 2 (A) Straightening of a mildly tortuous duct and gland during intraductal probing. The duct is noticeably tortuous in image before probing (black lines closely parallel the central ducts). (B) $4 \mathrm{~mm}$ probe has been advanced and is visualised within the duct tract. Note the duct and gland appear significantly straighter and the interglandular connective tissue area (arrows in A-C) seems reduced. (C) Immediately after probe withdrawal, persistent straightening of the proximal duct and gland. (D) At 2-month follow-up. The same gland with configuration of gland and interglandular tissue restored to near pre-probing appearance suggesting interglandular tissue is spongy, able to be compressed and re-expand allowing duct and gland flexibility.
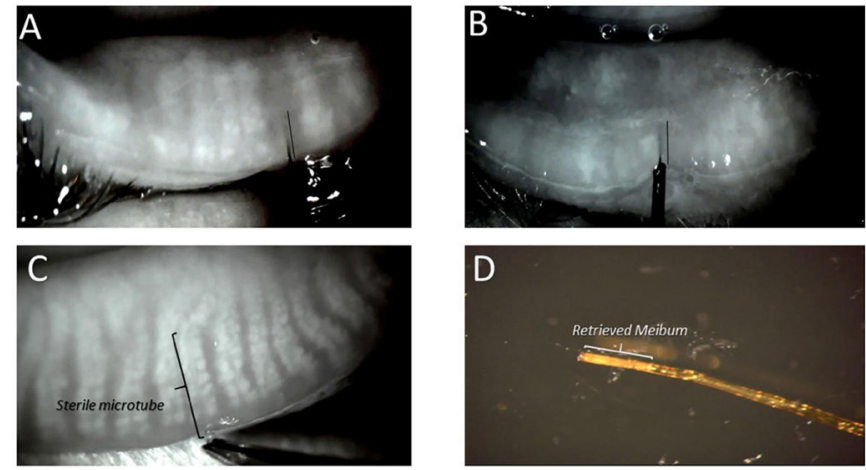

Figure 3 Four images where $(A)$ and $(B)$ demonstrate presence and location of intraductal devices (maskin probe) within the central duct of an area lacking acinar-ductule units. (A) Probe within the central duct of whole gland atrophy lacking acinar-ductule units for whole gland.

(B) Maskin probe within the central duct of proximal atrophy where the gland lacks acinar-ductule units proximal to areas of distal and midto-distal intact or faded acinar-ductule units (black lines parallel probe within central duct). (C) Microtube within the duct of a meibomian gland to retrieve meibum specimen. Note the forceps holding end of tube. (D) Microtube with retrieved meibum immediately after removal from gland.

among completely atrophied glands. Statistical significance level was set at $\mathrm{p}<0.05$.

\section{RESULTS}

\section{Visualisation of probe within intraductal space}

997 total gland orifices were identified (table 1). 996 gland orifices were probed from 38 lids using a $1 \mathrm{~mm}$ probe. 914 glands (probe visibility: 91.8\%) were visualised within the central duct, while 82 glands were probed through its orifice but not visualised inside the gland. The probe was determined to be within the central duct as acinar-ductule units were noted to surround the device (unless extensive acinar-ductule atrophy) including posteriorly with ease of device movement to and fro (figure 1A-C). Of 996 probed gland orifices, 139 (14\%) were observed to be WGAs and 857 (86\%) glands were non-WGAs. The movement of probes could be followed in real-time by viewing the image on the monitor. The duct and probe conformed to each other during probe passage. The central duct and gland were flexible in that the advancing probe appeared to affect a straightening of the central duct and proximal gland from inside the gland without affecting the neighbouring gland but could be seen to reduce the interglandular connective tissue space (figure 2). A similar straightening effect on gland morphology was noted through external manipulation of the hold on the lower lid. ${ }^{22}$ Meibography guided probing by following the device location viewed on the monitor ensured safe location and enabled selective localisation of device tip. There were no false passages. In some cases, visualisation of the entire length of probe was limited in areas of increased posterior acinar-ductule gland density. Figure 3 A,B shows successful advancement of probe into central duct in cases of WGA and proximal atrophy.

\section{Correlation of probe findings to WGA on meibography}

139 of 140 glands with WGA (99.3\%) showed a patent central duct to a $1 \mathrm{~mm}$ probe. The cumulative gland data for all WGAs from 24 lids combined showed probe findings of $106(76 \%)$ glands with FFFUR, 21 (15\%) with SFT and 12 (9\%) with NR We also looked at frequency of NR in WGA glands for each of 24 
Table 2 Distribution of NR as a percentage of WGA and overall probe findings (mean) in a lid across three groups

\begin{tabular}{|c|c|c|c|c|c|c|c|}
\hline Group & \#WGA (sum) & \#NR (within WGA) (sum) & $\%$ NR (within WGA) (mean) & \%FFFUR & $\%$ SFT & $\% N R$ & Total glands \\
\hline Group 1 (0 WGA) & 0 & 0 & NA & $61.9^{*}$ & 24.4 & $13.7+$ & 334 \\
\hline Group 2 (1-4 WGA) & 23 & 0 & $0 \%$ & 82.1 & 16.2 & 1.7 & 390 \\
\hline Group 3 ( $\geq 5$ WGA) & 116 & 12 (range: $0-5$ ) & $9.4 \% \ddagger$ (range: $0 \%-38.5 \%$ ) & 77.1 & 17.1 & 5.8 & 272 \\
\hline Total (38) & 139 & 12 & & & & & 996 \\
\hline
\end{tabular}

*Significantly lower compared with group $2(p=0.005)$ as per Mann-Whitney U test. tSignificantly higher compared with group $2(p=0.011)$ as per Mann-Whitney $U$ test. \#Significantly higher compared with group $2(\mathrm{p}=0.005)$ as per Mann-Whitney $\mathrm{U}$ test.

FFFUR, firm, fixed, focal and unyielding resistance; NA, not applicable; NR, no resistance; SFT, soft resistance; WGA, whole gland atrophy.

lids. We noted group 3 lids ( $\geq 5 \mathrm{WGA} / \mathrm{lid}$ ) to have higher mean frequency of NR among glands with WGA when compared with group $2(1-4 \mathrm{WGA} / \mathrm{lid})(\mathrm{p}=0.005)$ by Mann-Whitney $\mathrm{U}$ test (table 2).

\section{Incidence of probe findings in lids with WGA and non-WGA glands}

There was no statistical difference in the distribution of probe findings among the cumulative total numbers of non-WGA and WGA glands from all 38 lids. Distribution of probe findings (FFFUR, SFT and NR) for total glands probed within each lid (WGA plus Non-WGA) was also calculated. We noted a statistically significant increased mean NR per total glands probed in a lid for group 1 (0 WGA/lid) lids compared with group 2 $(p=0.011)$ by Mann-Whitney $U$ test (table 2$)$. There was a corresponding statistically significant decrease in \% FFFUR for group 1 lids compared with group 2 lids $(\mathrm{p}=0.005)$ by Mann-Whitney U test.

\section{Intraductal microtube}

Under real-time IR video feed, injection of intraductal dexamethasone using stainless steel microtube (figure 1D) showed no frank distention of gland structures. Placement of intraductal polyimide microtube (figure $3 \mathrm{C}$ ) enabled retrieval of meibum specimen (figure $3 \mathrm{D}$ ).

\section{DISCUSSION}

Our ability to safely cannulate and treat the Meibomian gland from the inside broadens the range of tissues and organs that are internally cannulated in routine medical practice at the beginning of the 21st century. These include exocrine salivary glands as well as pancreas and biliary tree, in addition to cerebral, coronary and renal arteries, plus the urethra and oesophagus for treatment of strictures. For urethral and esophageal lumen strictures, the internal approach to dilating the lumen is an effective targeted treatment of mechanical blockage. We have found the same to be true of treating internal strictures of the Meibomian glands with intraductal probing. By visualising over $90 \%$ of the probes within the intraductal space of lower lid glands, this study confirms the safety of probing and with meibography guided probing can bring added safety in the setting of excessively tortuous glands within the lower lid. There were no false passages noted which has been consistent with our earlier clinical experience. The novel imaging procedure presented earlier is essential in providing further understanding to the intraductal space. Clinicians and researchers will be able to correlate the tactile and auditory probe findings with visual meibography appearance. It will enable the localisation of intraductal devices. Localisation provides a diagnostic role as it may reveal location and extent of occult fixed resistance with compromise of ductal integrity as well as enable selective specimen retrieval.
Therapeutically, this imaging procedure may demonstrate relief of fixed resistance with restored ductal integrity, as well as enable injection of therapeutics to selective areas within the intraductal space. Also, with restored central duct integrity it is feasible to place a meibography directed stent for future selective treatment of WGA or proximal atrophy. The stent may retain the newly established integrity of the central duct during efforts to grow gland tissue. These imaging techniques for intraductal procedures may be of significant value for gland rehabilitation. Also, new users of this technology may benefit during initial skill acquisition.

With use of this technology, we were able to correlate probe findings of the intraductal space with meibography appearance. We found that the central duct channel persisted or was restored through probing in nearly all cases of WGA (with orifices) with the $1 \mathrm{~mm}$ probe as well as in proximal atrophic segments of truncated, short glands using 2 and $4 \mathrm{~mm}$ probes to reach the proximal duct with associated acinar-ductule atrophy. These findings were consistent with my (SLM) earlier findings during development of an in vitro culture system for rabbit meibomian gland where surgical and enzyme digestion to isolate the gland lead to loss of acinar-ductule units but the central duct proved relatively durable. ${ }^{2324}$ This exciting discovery reveals the patency of the central duct may persist or be restored in spite of pathology leading to acinar-ductule atrophy. This study also showed the majority of WGA had fixed resistance in $76 \%$ of these cases using a $1 \mathrm{~mm}$ probe. As over three quarters of gland showing WGA had fixed resistance indicating periductal fibroses, it is interesting to hypothesise whether periductal fibroses is related to WGA. Naturally, tight bands of periductal fibroses could elevate IDP leading to lid tenderness and ultimately gland atrophy. However, there may be an additional dynamic at work. Figure 1 from our recent paper $^{8}$ revealing probe findings within the intraductal space showed confocal microscopy examples of periductal fibrosis invading the external duct wall in association with scalloping and distortion of duct wall, as well as lumen channel contraction and stricture with overall loss of ductal integrity. We have also seen fibrovascular duct wall invasion and now believe that these reactions targeting the exterior duct wall and perhaps epithelial basement membrane may represent a loss of meibomian gland stem cells which may manifest with acinar-ductule atrophy. As research has now shown, in a mouse model of MGD, that regeneration of acinar-ductule units and the re-establishment of a working gland requires ductal integrity, we believe it will be clinically important to confirm or establish patency for the central duct in areas of acinar-ductule atrophy. ${ }^{25}$ This key concept of the necessity for ductal integrity for gland health and regrowth is supported by our 2018 paper in the British Journal of Ophthalmology showing, in a retrospective study, over $40 \%$ of upper lids with signs of gland regrowth after probing. ${ }^{11}$ Still, $24 \%$ of WGA did not show fixed resistance with the $1 \mathrm{~mm}$ probe 
which may represent an earlier stage of fibroses with external duct wall invasion but without development of a tight periductal fibrotic band within this depth. Also interesting is the observation that $73 \%$ of non-WGA cases showed fixed obstruction suggesting that FFFUR is a common probe finding in meibomian gland dysfunction, ${ }^{8}$ indicating a risk for future gland atrophy. This risk for future gland atrophy, from elevated IDP or possibly stem cell loss within the ductal epithelium, suggests the need to be pro-active and restore ductal integrity in patients with MGD, a disease well known to progress in a subclinical manner. ${ }^{626}$ For these reasons and from the lack of adverse sequelae reported in the published peer reviewed literature as well as after personally probing more than 100000 glands, I (SLM) have developed an initial therapeutic approach toward obstructive MGD focusing on early intervention with gland probing. This early intervention would serve to confirm or restore ductal integrity through the release of fixed obstructions from periductal fibrotic bands. Indications include MGD patients who are symptomatic as well as those who are asymptomatic with subclinical disease but with signs of MGD at the slit lamp or on IR meibography. ${ }^{27}$ Lids showing minimal disease such as non-obvious MGD as well as more moderate and severe disease are all probed to release periductal fibrosis and confirm or restore ductal integrity. A more complete discussion of probing indications has been published. ${ }^{9}$ Glands are probed annually to maintain intraductal integrity. Patients to consider earlier for re-probing are those with MGD presenting for cataract or refractive surgery as well as those with significant comorbidities, which increase the incidence and severity of obstructive MGD with fixed resistance. Additional MGD patients in whom to consider earlier re-probing include those with delayed tear clearance, a history of exposure to particulate matter, long-term use of eye cosmetics, chronic topical glaucoma therapy and those with tarsal inflammation such as from graft-versus-host disease, chemical and thermal injury and systemic autoimmune disease. ${ }^{9}$

The approach to using gland probing as first intervention for MGD not responding to conventional therapy of warm compresses, massage and artificial tears was evaluated in a recently published randomised controlled trial. ${ }^{28}$ Researchers found best clinical results with statistically significant improvement in standardised patient evaluation of eye dryness scores, tear break up time, meibum grade and lid telangiectasia using probing as initial therapy to physically release fixed obstructions and restore ductal integrity followed by subsequent IPL to 'inhibit telangiectasia' as compared with probing or IPL alone. Gland probing alone was found to provide statistically significant improvement in lid tenderness when compared with intense pulsed light (IPL) alone $(\mathrm{p}<0.001)$. For the IPL alone group, as the authors have noted, nearly $15 \%$ of patients showed 'even more serious symptoms at the end of the IPL treatment course', likely due to persistence of fixed obstruction. The authors concluded that the 'heat released by IPL and the pressure caused by the forceps might paradoxically increase the intraductal pressure and exacerbate the inflammatory response; thus, treatment with IPL alone may not alleviate disease symptoms but instead irritate the condition'. Taken together, these data provide additional support that probing is uniquely able to provide positive physical proof of release of intraductal fixed obstruction with equilibration of IDPs and resolution of lid tenderness. Furthermore, in our opinion, the authors' results using gland probing would show even greater improvement with added patient comfort by using Maskin probes and tubes which have been specially designed and sized for the meibomian glands, in addition to jojoba anaesthetic topical ointment. For example, our probes and tubes are 76 and 104110 microns in diameter, respectively, significantly smaller than the probes and tubes measuring 120 and 160 microns in diameter, respectively, that were used in this randomised controlled trial. I (SLM) also always start my probing by using our $1 \mathrm{~mm}$ long probe, the shortest and stiffest, to consistently find the entry angle into each gland, while using longer probes sequentially if clinically indicated. Starting with longer probes before a communication is established with the intraductal space, such as in this study, may lead to probe buckling and be more difficult to consistently find the duct entry angle. A longer probe that is also larger, may not buckle however, may be more traumatic and less forgiving.

Regarding efficacy of gland probing related to MGD severity, we have reported clinical data for lids with grades 3 or 4 atrophy as well as grades 1 or 2 that showed statistically significant improvement in signs and symptoms of o-MGD for both groups including lid tenderness, lid functionality as a meibum secreting unit and the number of expressible glands. ${ }^{11}$ Although there was no statistically significant difference between the two sets of grades, grades 1 and 2 had better results at each time point. Collectively, for lid tenderness, a total of 541 tender lids were probed. By 1-week, 3-6 months and 1-year follow-up, 86.1\% of $144,82.3 \%$ of $215 \%$ and $58.4 \%$ of 113 tender lids probed remained non-tender, respectively. In addition, there was a total of 271 non-functional lids (with four or less expressible glands per lid) that were probed. By 1-week, 3-6 months and 1-year follow-up, $93.8 \%$ of $64,92.2 \%$ of $102 \%$ and $73.9 \%$ of 46 non-functional lids remained functional, respectively. In this cohort of probed, non-functional lids, the preprobing average of expressible glands was $2.3 \pm 1.5$ per lid (counting only up to 10 expressible glands per lid). By 1-week, 3-6 months and 1 -year postprobing follow-up, there was a marked improvement in the number of expressible glands per lid to $8.7 \pm 2.1$ (264\% increase, $p<0.0001), 8.5 \pm 2.2(253 \%$ increase, $p<0.0001)$ and $7.0 \pm 3.0$ (193\% increase, $\mathrm{p}<0.0001)$, respectively. (It should be noted that a later study looked at the total number of expressible glands counted before and after probing, and did not stop at 10 expressible glands. In 25 preprobing non-functional lids, the average number of expressible glands was $2.8 \pm 1.1$ glands per lid, with a significant increase in the number of postprobing expressible glands per lid to $14.4 \pm 6.4(412 \%$ increase, $\mathrm{p}<0.0001)$ at a mean follow-up of 2.4 months.)

It had always been my (SLM) impression that when the probe was advanced through the orifice and into the distal duct that the probe follows the contour of the central duct as an arm would follow a sleeve, thereby assuring a safe passage and preventing false passages. Now with visualisation of the probing procedure in real time it becomes clear that while advancing the probe, the probe and duct conform to each other. In other words, the duct is able to alter its 3 -dimensional conformation and straighten as the probe advances. As the central duct straightens, the gland likewise straightens, but does not impact the neighbouring gland while the interglandular space narrows. This finding suggests that the central duct and gland are not rigid structures within the lid but rather have flexibility and df degrees of freedom of movement while the interglandular space appears spongy able to compress and re-expand. This makes sense intuitively as the gland routinely must conform to any manipulation of the lid from rubbing, pulling or other mechanical trauma as well as tissue oedema exerting external pressure on the gland from a neighbouring hordeolum. The gland must flex and deform as long as it avoids being engulfed in the inflammation. We have noted and reported on similar effects on gland morphology with external lid manipulation, ${ }^{22}$ particularly of the lower lid. The relevant 
unique observation is the ability to safely exert such an effect using an instrument from inside the central duct. Here we see another example of the relative flexibility, durability and strength of the central duct in addition to what we have described earlier where it survives contemporaneously to progressive whole gland acinar-ductule atrophy. This gland flexibility may be related to the relative health of the periglandular tissue such that oedema or inflammatory enzyme weakening of this surrounding tissue may lead, respectively, to limitation of or, conversely, increased potential movement of the gland inside the lid. Furthermore, perhaps these dynamic forces could lead to gland tortuosity. As the distal meibomian glands are anchored at the lid margin, ${ }^{9} 10$ with weakening of periglandular tissue, gravity may induce more proximal portions of glands to undergo progressive microscopic migration often seen in upper lids where gravity would have a greater impact. Increased gland flexibility with enzyme inflammatory weakening of interglandular tissue may lead to increased tortuosity with possible decreased meibum delivery and possible duct dilation as the duct may expand without strong interglandular tissue. Alternatively, in the setting of periglandular oedema there may be decreased gland flexibility from lid congestion with resultant reduced gland functionality. Decreased flexibility from stiffer, less spongy interglandular tissue may lead to reduced delivery of meibum with subsequent stasis and increased inflammation.

Our data suggested a bimodal profile for NR. There was an increase in NR relative to WGA in group 3 (with $\geq 5 \mathrm{WGA}$ / lid) when compared with group 2 (1-4 WGA/lid). This may reflect a more advanced atrophic gland and lid disease with possible weakened periglandular tissue enabling the ductal tissue to microdilate. Another possibility is a reduction in the rigidity of the duct wall. We also saw an increased NR relative to total glands probed in group 1 (with zero WGA) along with decreased FFFUR when compared with group 2 (1-4 WGA/lid). This finding may reflect less diseased gland ducts in group 1 lids.

Insertion of an intraductal microtube was also visualised in real-time and showed no distortion or distention of gland structures with injection of an estimated $8 \mu \mathrm{l}$ of a solution of dexamethasone. This would suggest a lack of intrinsic distensibility of glandular structures. This would further indicate that cystic deformation may not be an acute change from elevated IDP but require chronicity of elevated IDP unless in the presence of concomitant weakening of periglandular connective or glandular structural tissue such as in the setting of significant inflammation.

We have also showed the ability to retrieve meibum specimen from inside the intraductal space under direct observation. Strategies can therefore be implemented to selectively retrieve meibum from localised proximal or distal space as well as from either side of a fixed obstruction.

Limitations of this procedure at this time include a difficulty visualising the upper lid. This limitation is based on ergonomic and visualisation needs of being able to see the orifice at the same time the lid is everted. For the upper lid, this would require entering the orifice from a vertical position above the everted lid which would be challenging. However, this technology may be reconfigured for a procedure room operating microscope. By having the patient flat and supine, the everted upper lid orifices would be well visualised. This approach would also have a fixed focal length and eliminate the need to use one hand to operate the slit lamp joy stick; therefore, both hands are dedicated to positioning and probing the glands. A limitation of this study may include possibility of pseudoreplication where observations on consecutive glands may be influenced on by the previous gland.
Another potential limitation of this procedure is the probing learning curve. The curve however is shallow and following recommendations, such as obtaining adequate anaesthesia with JAO and always beginning with the $1 \mathrm{~mm}$ probe (which is the shortest and stiffest), leads to a successful and safe procedure. Indeed, there are now 11 published independent manuscripts in the peer-reviewed literature without report of adverse sequelae.

The ability to perform meibography guided intraductal probing and visualise these procedures in real-time brings new excitement to the future of MG research. By confirming the safe intraductal insertion of devices, the exploration of novel targeted approaches to MG therapies has entered a new era; at the same time research has shown that a key requirement for acinar regeneration is ductal integrity. The retention of a duct channel in the setting of WGA (with an intact orifice) as well as the atrophic acinar-ductule portions of glands with proximal atrophy, and our ability to restore ductal integrity using probing techniques suggest the possibility to prospectively utilise this ductal tissue for reconstructive and regenerative interventions. For those interested in treating MGD, we have taken a giant step forward.

Contributors SLM and SA planned and conducted the study, analysed the data as well as drafted and revised manuscript. SA performed statistical analysis. SLM is the guarantor.

Funding The authors have not declared a specific grant for this research from any funding agency in the public, commercial or not-for-profit sectors.

Competing interests SM is the owner of MGD Innovations, Inc which holds patents on instrumentation and methods for intraductal diagnosis and treatment of meibomian gland disease (MGD), plus related pending patents including visualisation of real-time intraductal diagnostics, probing and other therapies. SM also has patents on the use of jojoba-based treatment options for MGD.

Patient consent for publication Not required.

Provenance and peer review Not commissioned; externally peer reviewed Data availability statement All data relevant to the study are included in the article or uploaded as supplementary information.

Open access This is an open access article distributed in accordance with the Creative Commons Attribution Non Commercial (CC BY-NC 4.0) license, which permits others to distribute, remix, adapt, build upon this work non-commercially, and license their derivative works on different terms, provided the original work is properly cited, appropriate credit is given, any changes made indicated, and the use is non-commercial. See: http://creativecommons.org/licenses/by-nc/4.0/.

\section{ORCID iD}

Steven L Maskin http://orcid.org/0000-0003-4692-7787

\section{REFERENCES}

1 Nichols KK, Foulks GN, Bron AJ, et al. The International workshop on meibomian gland dysfunction: Executive summary. Invest Ophthalmol Vis Sci 2011;52:1922-9.

2 Lemp MA, Crews LA, Bron AJ, et al. Distribution of aqueous-deficient and evaporative dry eye in a clinic-based patient cohort: a retrospective study. Cornea 2012;31:472-8.

3 Cher I. Meibomian marginal dimples: clinical indicants of reactive pathogenic processes. In: Lass J, ed. Advances in corneal research: selected transections of the world Congress on the cornea. New York: Plenum Press, 1997: 27-35

4 Foulks GN, Bron AJ. Meibomian gland dysfunction: a clinical scheme for description, diagnosis, classification, and grading. Ocul Surf 2003;1:107-26.

5 Matsumoto Y, Sato EA, Ibrahim OMA, et al. The application of in vivo laser confocal microscopy to the diagnosis and evaluation of meibomian gland dysfunction. Mo/ Vis 2008;14:1263-71.

6 Maskin SL. Intraductal meibomian gland probing relieves symptoms of obstructive meibomian gland dysfunction. Cornea 2010;29:1145-52.

7 Maskin SL. Meibomian gland probing findings suggest fibrotic obstruction is a major cause of obstructive meibomian gland dysfunction (O-MGD). IOVS 2012;53:1.

8 Maskin SL, Alluri S. Expressible meibomian glands have occult fixed obstructions: findings from meibomian gland probing to restore intraductal integrity. Cornea 2019:38:880-7.

9 Maskin SL, Alluri S. Intraductal meibomian gland probing: background, patient selection, procedure, and perspectives. Clin Ophthalmol 2019;13:1203-23.

10 Maskin SL. Intraductal Meibomian Gland Probing: A paradigm shift for the successful treatment of obstructive meibomian gland dysfunction. In: Tsubota K, ed. Diagnosis 
and treatment of meibomian gland dysfunction. Tokyo, Japan: Kanehara \& Co.Ltd, 2016: 149-67.

11 Maskin SL, Testa WR. Growth of meibomian gland tissue after intraductal meibomian gland probing in patients with obstructive meibomian gland dysfunction. $\mathrm{Br} J$ Ophthalmol 2018;102:59-68.

12 Fermon S, Zaga IH, Alvarez Melloni D. [Intraductal meibomian gland probing for the treatment of blepharitis]. Arch Soc Esp Oftalmol 2015;90:76-80.

13 Dongju Q, Hui L, Jianjiang X. Clinical research on intraductal meibomian gland probing in the treatment of patients with meibomian gland dysfunction. Chin J Optom Ophthalmol 2014;16:615-21.

14 Nakayama N, Kawashima M, Kaido M, et al. Analysis of Meibum before and after intraductal meibomian gland probing in eyes with obstructive meibomian gland dysfunction. Cornea 2015:34:1206-8.

15 Ma X, Lu Y. Efficacy of intraductal meibomian gland probing on tear function in patients with obstructive meibomian gland dysfunction. Cornea 2016;35:725-30.

16 Sik Sarman Z, Cucen B, Yuksel N, et al. Effectiveness of intraductal meibomian gland probing for obstructive meibomian gland dysfunction. Cornea 2016;35:721-4.

17 Syed ZA, Sutula FC. Dynamic intraductal meibomian probing: a modified approach to the treatment of obstructive meibomian gland dysfunction. Ophthalmic Plast Reconstr Surg 2017;33:307-9.

18 Wladis EJ. Intraductal meibomian gland probing in the management of ocular rosacea. Ophthalmic Plast Reconstr Surg 2012;28:416-8.

19 Díaz TC, Izquierdo DC, López IH, et al. Permeabilizing probing for the treatment of obstructive meibonian gland dysfunction. Revista Cubana de Oftalmología 2014;27:301-10.
20 Incekalan TK, Harbiyeli II, Yagmur M, et al. Effectiveness of intraductal meibomian gland probing in addition to the conventional treatment in patients with obstructive meibomian gland dysfunction. Ocul Immunol Inflamm 2019;27:1-7.

21 Nirupama D, Hymavathi B, Prathima L, et al. Meibomian gland probing in patients with meibomian gland dysfunction. IJCEO 2019;5:78-81.

22 Maskin SL, Testa WR. Infrared video Meibography of lower lid meibomian glands shows easily distorted glands: implications for longitudinal assessment of atrophy or growth using lower lid Meibography. Cornea 2018;37:1279-86.

23 Maskin SL, Tseng SC. Culture of rabbit meibomian gland using collagen gel. Invest Ophthalmol Vis Sci 1991;32:214-23.

24 Maskin SL, Tseng SC. Clonal growth and differentiation of rabbit meibomian gland epithelium in serum-free culture: differential modulation by EGF and FGF. Invest Ophthalmol Vis Sci 1992;33:205-17.

25 Reneker LW, Yang X, Zhong X, et al. Meibomian gland (Mg) acinar regeneration from atrophy in a FGFR2 conditional knockout mouse model. Investig Ophthalmol Vis Sci 2019;60:1412.

26 Maskin SL, Greenberg EL. Meibomian gland dysfunction, dry eye disease, and Unhappy cataract surgery patients. Cataract \& Refractive Surgery Today 2018:15-17.

27 Arita R, Itoh K, Maeda S, et al. A newly developed noninvasive and mobile penshaped meibography system. Cornea 2013;32:242-7.

28 Huang X, Qin Q, Wang L, et al. Clinical results of intraductal meibomian gland probing combined with intense pulsed light in treating patients with refractory obstructive meibomian gland dysfunction: a randomized controlled trial. BMC Ophthalmol 2019;19:211. 